\title{
Article \\ New BBP-Type Formulae for $\pi$ Derived from New Forms of Taylor Expansions of Inverse Tangent Function
}

\author{
Xin Wu*, Zhongfan Chen and Yinan Zhu
}

Citation: $\mathrm{Wu}, \mathrm{X}$.; Chen, Z.; Zhu, Y. New BBP-Type Formulae for $\pi$ Derived from New Forms of Taylor Expansions of Inverse Tangent Function. Mathematics 2022, 10, 290 https: / / doi.org/10.3390/ math10030290

Academic Editors: Jan L. Cieśliński and Ioannis Argyros

Received: 1 November 2021

Accepted: 14 January 2022

Published: 18 January 2022

Publisher's Note: MDPI stays neutral with regard to jurisdictional claims in published maps and institutional affiliations.

Copyright: (C) 2022 by the authors. Licensee MDPI, Basel, Switzerland. This article is an open access article distributed under the terms and conditions of the Creative Commons Attribution (CC BY) license (https:// creativecommons.org/licenses/by/ $4.0 /)$.
School of Civil Engineering, Southeast University, Nanjing 211189, China; 220191052@seu.edu.cn (Z.C.); yinan_zhu@seu.edu.cn (Y.Z.)

* Correspondence: 220191224@seu.edu.cn

\begin{abstract}
In this work, we give two new Taylor expansions of $\arctan (x+\omega)$, where $\omega$ represents a finite increment of $x$. We discover several remarkable infinite series from these expansions by special substitutions. Some of these infinite series give BBP-type formulae.
\end{abstract}

Keywords: BBP-type formula; Taylor expansion; infinite series

\section{Introduction}

In 1673 , Leibniz obtained an elegant infinite series of $\pi / 4$ that now bears his name [1], since then this field of mathematic began to attract widespread attentions among mathematicians. Leibniz's work was primarily concerned with quadrature, the $\pi / 4$ series resulted when he applied his method to the circle [2]. Later, Euler made the fundamental contributions to the theory of infinite series. He discovered a large number of infinite series [2-7]; these series give formulas for $\pi$ as numerical results. Euler's method of analysis has greatly improved the development of this branch of mathematics.

In 1997, David Bailey, Peter Borwein, and Simon Plouffe discovered a striking formula for $\pi$ [8], and it can be used to generate the nth base-16 digit of $\pi$ without having to look at any prior digit [9]. A formula of this type is now called "BBP-type formula". After that, many researchers denoted themselves into this field; Chan [10] gave BBP-type formulas involving the golden ratio; Adegoke [11,12], Bailey-Borweins-Plouffe [13,14], Chan [15,16], Wei [17], Zhang [18,19], Guillera [20], and Takahashi [21-23] have found many new BBPtype formulas.

The BBP-type formula was originally discovered by numerical methods; the authors first supposed that such a formula exists and then searched for it using high-precision approximate reals, a high-performance SGI workstation, and the PSLQ algorithm. Many researchers obtained new formulas by this method [9,12-14,23-25]; it is worthwhile to note that Takahashi [22] proposed a method of using Montgomery multiplication in the computation of a binary BBP-type formula. However, this is not the only method to find the BBP-type formula. Indeed, the numerical method is not priori because it can provide only the formula but not the origin of the formula. For this reason, when a BBP-type formula is discovered via an algorithm, we still need to provide a mathematical proof to make the result rigorous. The second method to find a BBP-type formula is based on the theory of calculus and infinite series. In this method, we need to firstly find infinite series, and their numerical results are BBP-type formulas. It can be clearly seen that these infinite series are the real origin of BBP-type formulas, and thus the method is priori. Some researchers find new formulas by this method; Adegoke [11], Wei [17], and Chan [10,15,16] gave new BBP-type formulas by integral calculus, with some of the formulas involving the golden ratio; Zhang [18] discovered many new BBP-type formulas with different moduli by the expansion of $\arctan [a /(x-1)]$; Bailey [26] made a systematic summary of the origin and development of BBP-type formulas. It is especially worthwhile to note that the inverse tangent function is closely related to the second method, useful infinite series can be 
obtained from the study of it [18]. Indeed, the well-known Gregory series can be seen as the simplest series of this type, and it is the infinite series of the inverse tangent function.

In this paper, we will give two new Taylor's expansions of $\arctan (x+\omega)$. These expansions have clear structures and certain laws of the progressions. We find several new infinite series from these expansions, and their numerical results are BBP-type formulas. It is worthwhile to note that Gregory's series and Leibniz's formula for $\pi$ can also be deduced from the new expansions. In addition, we also find other infinite series; although they can not provide new BBP-type formulas, they are remarkable for their simplicity and regularity.

\section{New Taylor Expansion of $\arctan (x+\omega)$}

Let $y=\arctan x$, if $x$ changes by a finite increment $\omega$, according to the well-known Taylor's expansion, we can represent the value of $y$ at $x+\omega$ as

$$
\arctan (x+\omega)=\arctan x+\sum_{n=1}^{\infty} \frac{\omega^{n} d^{n} y}{d x^{n}}
$$

Since $y=\arctan x$, we have

$$
\begin{aligned}
& \frac{d y}{d x}=\frac{1}{1+x^{2}}, \frac{d^{2} y}{d x^{2}}=\frac{-2 x}{\left(1+x^{2}\right)^{2}}, \frac{d^{3} y}{d x^{3}}=\frac{6 x^{2}-2}{\left(1+x^{2}\right)^{3}}, \frac{d^{4} y}{d x^{4}}=\frac{-24 x^{3}+24 x}{\left(1+x^{2}\right)^{4}} \\
& \frac{d^{5} y}{d x^{5}}=\frac{120 x^{4}-240 x^{2}+24}{\left(1+x^{2}\right)^{4}}, \ldots ;
\end{aligned}
$$

Hence,

$$
\arctan (x+\omega)=\arctan x+\frac{\omega}{1+x^{2}}-\frac{\omega^{2} x}{\left(1+x^{2}\right)^{2}}+\frac{\omega^{3}\left(x^{2}-1 / 3\right)}{\left(1+x^{2}\right)^{3}}-\frac{\omega^{4}\left(x^{3}-x\right)}{\left(1+x^{2}\right)^{4}}+\ldots
$$

This series, since the expression has no certain law, can provide nothing valuable to us. Nevertheless, it can be transformed into another form, whose structure is immediately clear. This new expansion can be suitably seen as a theorem, and, for this aim, we need to firstly introduce a lemma.

Lemma 1. Suppose $u=\pi / 2-\arctan x, y=\arctan x$, then

$$
\frac{d^{n} y}{d x^{n}}=(-1)^{n-1}(n-1) ! \sin ^{n} u \sin n u
$$

Here, $n \in N^{+}$.

Proof. Since $u=\pi / 2-\arctan x$, i.e., $x=\cot u$, it will be

$$
\frac{d y}{d x}=\frac{1}{1+x^{2}}=\sin ^{2} u
$$

or

$$
d y=\sin ^{2} u d x
$$

Take the differential of the formula, and we have

$$
d^{2} y=\sin 2 u d u d x
$$

since $x=\cot u, d u=-\sin ^{2} u d x$, substitute the expression for $d u$ in this formula, and it will be

$$
d^{2} y=-\sin ^{2} u \sin 2 u d x^{2}
$$

Take the differential of this formula and substitute $d u$ for $-\sin ^{2} u d x$, and it will be

$$
d^{3} y=2 \sin ^{3} u \sin 3 u d x^{3} .
$$


Similarly, continuing for higher differentials of $y$, we have

$$
\begin{aligned}
& \frac{d y}{d x}=+\sin u \sin u, \\
& \frac{d^{2} y}{d x^{2}}=-\sin ^{2} u \sin 2 u, \\
& \frac{d^{3} y}{d x^{3}}=+2 ! \sin ^{3} u \sin 3 u, \\
& \frac{d^{4} y}{d x^{4}}=-3 ! \sin ^{4} u \sin 4 u, \\
& \frac{d^{5} y}{d x^{5}}=+4 ! \sin ^{5} u \sin 5 u, \\
& \frac{d^{6} y}{d x^{6}}=-5 ! \sin ^{6} u \sin 6 u, \\
& \cdots
\end{aligned}
$$

From this, it can be easily seen that the proof of lemma is based on induction.

For $n=1$, the lemma is apparently correct.

Supposing

$$
\frac{d^{n} y}{d x^{n}}=(-1)^{n-1}(n-1) ! \sin ^{n} u \sin n u,
$$

we just need to differentiate this formula to determine $d^{\mathrm{n}+1} y / d x^{\mathrm{n}+1}$, and it will be

$$
\frac{d^{n+1} y}{d x^{n}}= \pm n ! \sin ^{n-1} u \sin (n+1) u d u ，
$$

substituting $d u$ for $-\sin ^{2} u d x$ in the expression, we have

$$
\frac{d^{n+1} y}{d x^{n+1}}=\mp n ! \sin ^{n+1} u \sin (n+1) u
$$

This finishes the proof.

By Lemma 1, substitute Formula (2) into Series (1), and we have the following theorem.

Theorem 1. Suppose $\omega$ is an arbitrary finite increment of the variable $x$, then

$$
\arctan (x+\omega)=\arctan x+\sum_{n=1}^{\infty} \frac{(-1)^{n-1} \omega^{n}}{n} \sin ^{n} u \sin n u
$$

\section{BBP-Type Formulas for $\pi$}

According to Theorem 1, we can obtain many remarkable corollaries, each corollary giving an infinite series and BBP-type formula for $\pi$. Firstly, it is worthwhile to note that the well-known Gregory series [1], and thus the Leibniz's Formula for $\pi$ [2] can also be deduced from this theorem. Thus, it can be seen as a new proof of this famous series and formula, as the following corollary.

Corollary 1. Suppose $\omega$ is an arbitrary finite quantity, then

$$
\arctan \omega=\sum_{n=0}^{\infty} \frac{(-1)^{n} \omega^{2 n+1}}{2 n+1}
$$

Here, $n \in N$.

Proof. In Theorem 1 , let $x=0$, then $\arctan 0=0, u=\pi / 2$, hence

$$
\sin u=1, \sin 2 u=0, \sin 3 u=-1, \sin 4 u=0, \ldots ;
$$


The sines of $n u$ take a cycle with a period 4; substituting these values in Series (3), we obtain Series (4).

In addition, this finishes the proof.

Remark 1. As it is known, this is the Gregory series [1], taking $\omega=1$ in it gives Leibniz's Formula for $\pi[2]$ :

$$
\frac{\pi}{4}=\sum_{n=0}^{\infty} \frac{(-1)^{n}}{2 n+1}
$$

Corollary 2. Suppose $\omega$ is an arbitrary finite quantity, then

$$
\begin{array}{r}
\arctan (1+\omega)=\frac{\pi}{4}+\frac{1}{2} \sum_{k=0}^{\infty} \frac{1}{2^{4 k}}\left(\frac{\omega^{8 k+1}}{8 k+1}-\frac{\omega^{8 k+2}}{8 k+2}+\frac{\omega^{8 k+3}}{2(8 k+3)}\right. \\
\left.-\frac{\omega^{8 k+5}}{2^{2}(8 k+5)}+\frac{\omega^{8 k+6}}{2^{2}(8 k+6)}-\frac{\omega^{8 k+7}}{2^{3}(8 k+7)}\right)
\end{array}
$$

Here, $k \in N$.

Proof. In Theorem 1 , let $x=1$, then $\arctan 1=\pi / 4, u=\pi / 4$, hence

$$
\begin{aligned}
& \sin u=\frac{1}{\sqrt{2}}, \sin 2 u=1, \sin 3 u=\frac{1}{\sqrt{2}}, \sin 4 u=0, \\
& \sin 5 u=-\frac{1}{\sqrt{2}}, \sin 6 u=-1, \sin 7 u=-\frac{1}{\sqrt{2}}, \sin 8 u=0, \ldots ;
\end{aligned}
$$

The sines of $n u$ take a cycle with a period 8 here; substituting these values in Series (3), we obtain Series (6).

In addition, this finishes the proof.

Remark 2. Put $\omega=-1$ in Series (6), and it will be

$$
\begin{aligned}
\frac{\pi}{4}= & \frac{1}{2} \sum_{k=0}^{\infty} \frac{1}{16^{k}}\left(\frac{1}{8 k+1}+\frac{1}{8 k+2}+\frac{1}{2(8 k+3)}\right. \\
& \left.-\frac{1}{2^{2}(8 k+5)}+\frac{1}{2^{2}(8 k+6)}-\frac{1}{2^{3}(8 k+7)}\right)
\end{aligned}
$$

This is a BBP-type formula, by the notation of P function introduced by Bailey [22]:

$$
P(s, b, m, A)=\sum_{k=0}^{\infty}\left(\frac{1}{b^{k}} \sum_{j=1}^{m} \frac{a_{j}}{(m k+j)^{s}}\right)
$$

Here, $s, b, m \in N^{+}$and $A=\left(a_{1}, a_{2}, \ldots, a_{j}\right)$, where $a_{i} \in N, i=1,2, \ldots, j$; this formula can be expressed as

$$
\frac{\pi}{4}=\frac{1}{2} P\left[1,16,8,\left(1,1,2^{-1}, 0,-2^{-2}, 2^{-2},-2^{3}, 0\right)\right]
$$

or

$$
\pi=P\left[1,16,8,\left(2,2,1,0,-2^{-1}, 2^{-1},-2^{-2}, 0\right)\right]
$$

Remark 3. The original BBP formula for $\pi$ found by Plouffe [8] is:

$$
\pi=P[1,16,8,(4,0,0,-2,-1,-1,0,0)]
$$

Thus, it can be clearly seen that Formula (10) converges more rapidly, the speed of convergence is determined only by $a_{j}$ in this case, and $a_{j}$ in Formula (10) are smaller. 
Remark 4. Series (6) can be expressed more concisely as

$$
\arctan (1+\omega)=\frac{\pi}{4}+\frac{1}{2} \sum_{k=0}^{\infty} \frac{\omega^{4 k+1}}{(-4)^{k}}\left(\frac{1}{4 k+1}-\frac{\omega}{4 k+2}+\frac{\omega^{2}}{2(4 k+3)}\right)
$$

Put $\omega=-1$ in this series, and we have

$$
\pi=\sum_{k=0}^{\infty} \frac{1}{(-4)^{k}}\left(\frac{2}{4 k+1}+\frac{2}{4 k+2}+\frac{1}{4 k+3}\right)
$$

In addition, it can be expressed by $P$ function as

$$
\pi=P[1,-4,4,(2,2,1,0)]
$$

This BBP-type formula was first discovered by Victor Adamchik and Stan Wagon via algorithm [9]. We discover this formula in an absolute different approach.

Remark 5. It is reasonable to name the series like (12) "BBP-type series", whose structure is exactly the same as the BBP-type formula. Indeed, Series (12) can be written as

$$
\arctan (1+\omega)=\frac{\pi}{4}+\frac{1}{2} \sum_{k=0}^{\infty} \frac{1}{(-4)^{k}}\left(\frac{\omega^{4 k+1}}{4 k+1}-\frac{\omega^{4 k+2}}{4 k+2}+\frac{\omega^{4 k+3}}{2(4 k+3)}\right)
$$

If we introduce a new function $W$ similar with function $P$ :

$$
W(\omega, s, b, m, A)=\sum_{k=0}^{\infty}\left(\frac{1}{b^{k}} \sum_{j=1}^{m} \frac{a_{j} \omega^{m k+j}}{(m k+j)^{s}}\right)
$$

Here $s, b, m \in N^{+}$and $A=\left(a_{1}, a_{2}, \ldots, a_{j}\right)$, where $a_{i} \in N, i=1,2, \ldots, j$, and $\omega$ is an arbitrary finite quantity; then, Series (12), which gives BBP-type Formula (14) as a numerical result, can be expressed now by $W$ function as

$$
\arctan (1+\omega)=\frac{\pi}{4}+\frac{1}{2} W[\omega, 1,-4,4,(1,-1,1,0)]
$$

Remark 6. It is worthwhile to note that, from this point of view, Gregory Series (4) is the simplest BBP-type series, and it can be expressed by Wunction as

$$
\arctan \omega=W[\omega, 1,2,-1,(1,0)]
$$

In addition, Leibniz's formula for $\pi$ is the simplest BBP-type formula:

$$
\frac{\pi}{4}=P[1,2,-1,(1,0)]
$$

According to the remarks of Corollary 2 above, especially the W function in Remark 5, we give the following corollaries of Theorem 1.

Corollary 3. Suppose $\omega$ is an arbitrary finite quantity, then

$$
\arctan (\sqrt{3}+\omega)=\frac{\pi}{3}+\frac{1}{4} W\left[\omega, 1,-64,6,\left(1,-\frac{\sqrt{3}}{2}, \frac{1}{2},-\frac{\sqrt{3}}{2^{3}}, \frac{1}{2^{4}}, 0\right)\right]
$$


Proof. In Theorem 1, put $x=\sqrt{3}$, then $\arctan \sqrt{3}=\pi / 3, u=\pi / 6$, hence

$$
\begin{aligned}
& \sin u=\frac{1}{2}, \sin 2 u=\frac{\sqrt{3}}{2}, \sin 3 u=1, \sin 4 u=\frac{\sqrt{3}}{2}, \sin 5 u=\frac{1}{2}, \sin 6 u=0, \\
& \sin 7 u=-\frac{1}{2}, \sin 8 u=-\frac{\sqrt{3}}{2}, \sin 9 u=-1, \sin 10 u=-\frac{\sqrt{3}}{2}, \sin 11 u=-\frac{1}{2}, \sin 12 u=0, \ldots ;
\end{aligned}
$$
we have

The sines of $n u$ take a cycle with a period 12 here; substituting these values in Series (3),

$$
\begin{aligned}
\arctan (\sqrt{3}+\omega) & =\frac{\pi}{3}+\frac{1}{4} \sum_{k=0}^{\infty} \frac{1}{2^{12 k}}\left(\frac{\omega^{12 k+1}}{12 k+1}-\frac{\sqrt{3} \omega^{12 k+2}}{12 k+2}+\frac{\omega^{12 k+3}}{2(12 k+3)}-\frac{\sqrt{3} \omega^{12 k+4}}{2^{3}(12 k+4)}\right. \\
& +\frac{\omega^{12 k+5}}{2^{4}(12 k+5)}-\frac{\omega^{12 k+7}}{2^{6}(12 k+7)}+\frac{\sqrt{3} \omega^{12 k+8}}{2^{7}(12 k+8)} \\
& \left.-\frac{\omega^{12 k+9}}{2^{7}(12 k+9)}+\frac{\sqrt{3} \omega^{12 k+10}}{2^{9}(12 k+10)}-\frac{\omega^{12 k+11}}{2^{10}(12 k+11)}\right)
\end{aligned}
$$

This series can be expressed more concisely as

$$
\arctan (\sqrt{3}+\omega)=\frac{\pi}{3}+\frac{1}{4} \sum_{k=0}^{\infty} \frac{1}{(-64)^{k}}\left[\frac{\omega^{6 k+1}}{6 k+1}-\frac{\sqrt{3} \omega^{6 k+2}}{2(6 k+2)}+\frac{\omega^{6 k+3}}{2(6 k+3)}-\frac{\sqrt{3} \omega^{6 k+4}}{2^{3}(6 k+4)}+\frac{\omega^{6 k+5}}{2^{4}(6 k+5)}\right]
$$

By the use of $W$ function, this series can be written as (20), and here the sequence $A=\left(a_{1}, a_{2}, \ldots, a_{j}\right)$ in $W$ function is not restricted in integers, but generalized to all real numbers, i.e., $a_{i} \in R, i=1,2, \ldots, j$.

This finishes the proof.

Remark 7. Put $\omega=-\sqrt{3}$ in Series (20), and we obtain a new BBP formula:

$$
\frac{\pi}{3 \sqrt{3}}=\sum_{k=0}^{\infty}\left(\frac{27}{-64}\right)^{k}\left[\frac{1}{2^{2}(6 k+1)}+\frac{3}{2^{2}(6 k+2)}+\frac{3}{2^{3}(6 k+3)}+\frac{3^{2}}{2^{5}(6 k+4)}+\frac{3^{2}}{2^{6}(6 k+5)}\right]
$$

This formula shows that $b$ in $\mathrm{P}$ function should be generalized from integers to rational numbers, i.e., $b \in Q$; then, Formula (23) can be written by P function as

$$
\frac{\pi}{3 \sqrt{3}}=P\left[1,-\frac{27}{64}, 6,\left(2^{-2}, 2^{-2}, 2^{-3}, 2^{-5}, 2^{-6}, 0\right)\right]
$$

It can be clearly seen that this BBP-type formula for $\pi$ converges much more rapidly than Formulas (10) and (11).

Corollary 4. Suppose $\omega$ is an arbitrary finite quantity, then

$$
\arctan \left(\frac{1}{\sqrt{3}}+\omega\right)=\frac{\pi}{6}+\frac{3}{4} W\left[\omega, 1,-\frac{3 \sqrt{3}}{8}, 3,\left(1,-\frac{\sqrt{3}}{2}, 0\right)\right]
$$

Proof. In Theorem 1, put $x=1 / \sqrt{3}$, then $\arctan 1 / \sqrt{3}=\pi / 6, u=\pi / 3$, thus

$$
\sin u=\frac{\sqrt{3}}{2}, \sin 2 u=\frac{\sqrt{3}}{2}, \sin 3 u=0, \sin 4 u=-\frac{\sqrt{3}}{2}, \sin 5 u=-\frac{\sqrt{3}}{2}, \sin 6 u=0, \ldots ;
$$

The sines of $n u$ take a cycle with a period 6 here; substituting these values in Series (3), we have

$$
\arctan \left(\frac{1}{\sqrt{3}}+\omega\right)=\frac{\pi}{6}+\frac{3}{4} \sum_{k=0}^{\infty}\left(\frac{\sqrt{3}}{2}\right)^{6 k}\left(\frac{\omega^{6 k+1}}{6 k+1}-\frac{\sqrt{3} \omega^{6 k+2}}{2(6 k+2)}+\frac{\sqrt{3}^{3} \omega^{6 k+3}}{2^{3}(6 k+4)}-\frac{\sqrt{3}^{4} \omega^{6 k+4}}{2^{4}(6 k+5)}\right)
$$


This series can be expressed more concisely as

$$
\arctan \left(\frac{1}{\sqrt{3}}+\omega\right)=\frac{\pi}{6}+\frac{3}{4} \sum_{k=0}^{\infty}\left(\frac{3 \sqrt{3}}{-8}\right)^{k}\left(\frac{\omega^{3 k+1}}{3 k+1}-\frac{\sqrt{3} \omega^{3 k+2}}{2(3 k+2)}\right)
$$

By the use of the $W$ function, it can be written as (25), and here $b$ in $W$ function is generalized to real numbers, i.e., $b \in R$.

In addition, this finishes the proof.

Remark 8. Generally, we conclude that it is convenient and necessary to generalize $b$ and $A$ in both $W$ function and $P$ function from integers to real numbers, i.e., $s, m \in N^{+}, b \in R$, and $A=\left(a_{1}, a_{2}, \ldots, a_{j}\right)$, where $a_{i} \in R, i=1,2, \ldots, j$.

Remark 9. Put $\omega=-1 / \sqrt{3}$ in Series (25), and we have another new BBP formula:

$$
\frac{\pi}{3 \sqrt{3}}=\sum_{k=0}^{\infty} \frac{1}{(-8)^{k}}\left[\frac{1}{2(3 k+1)}+\frac{1}{2^{2}(3 k+2)}\right]
$$

or

$$
\frac{\pi}{3 \sqrt{3}}=P\left[1,-8,3,\left(2^{-1}, 2^{-2}, 0\right)\right]
$$

\section{Infinite Series Deduced from Series (3)}

In the preceding section, we obtained several corollaries of Theorem 1, and each of them gave a BBP-type formula. In this section, nevertheless, we will give several corollaries that are different from the previous ones; these corollaries give new infinite series.

Corollary 5. Suppose $u \in(0, \pi)$, then

$$
\frac{\pi}{2}=u+\sum_{n=1}^{\infty} \frac{1}{n} \cos ^{n} u \sin n u
$$

Here, $n \in N$.

Proof. In Theorem 1, put $\omega=-x, \arctan (x+\omega)=0$, then we have

$$
\arctan x=\sum_{n=1}^{\infty} \frac{x^{n}}{n} \sin ^{n} u \sin n u
$$

Since

$$
\arctan x=\frac{\pi}{2}-u \text { and } x=\cot u=\frac{\cos u}{\sin u}
$$

Substitute these values in (31), and it will be

$$
\frac{\pi}{2}=u+\sum_{n=1}^{\infty} \frac{1}{n} \cos ^{n} u \sin n u
$$

This series is not valid for all values of $u$. Indeed, $y=\arctan x$ is a multi-valued function; for instance, $\arctan 1=\pi / 4$, but we also have $\arctan 1=9 \pi / 4,17 \pi / 4, \ldots$, which means, when the variable $x$ equals different values, the function $y$ can take the same value. This makes the differential calculus does not work, as it is known. Thus, we have to choose an interval of $y$ in which $y$ is a single-valued function, i.e., $y \in(-\pi / 2, \pi / 2)$. Furthermore, when we set $\omega=-x=0$ in Series (3), both sides of the equation vanish, whence the substitution loses its meaning in the derivation. Thus, $x \neq 0$, this also means $y \neq 0$ because $y=\arctan x$. Therefore, we limit $y$ in the interval $(0, \pi / 2)$; thus, series (30) are valid when 
$y \in(0, \pi / 2)$, which also means $u \in(0, \pi / 2)$ because $u=\pi / 2-y$. In addition, the equation retains the same when we substitute $u$ for $\pi-u$, for

$$
\frac{1}{n} \cos ^{n}(\pi-u) \sin (n \pi-n u)=\frac{1}{n}\left(-\cos ^{n} u\right) \sin n u=-\frac{1}{n} \cos ^{n} u \sin n u,
$$

when $n$ are odd numbers; and

$$
\frac{1}{n} \cos ^{n}(\pi-u) \sin (n \pi-n u)=\frac{1}{n} \cos ^{n} u(-\sin n u)=-\frac{1}{n} \cos ^{n} u \sin n u,
$$

when $n$ are even numbers. Thus,

$$
\frac{1}{n} \cos ^{n}(\pi-u) \sin (n \pi-n u)=-\frac{1}{n} \cos ^{n} u \sin n u, n \in N^{+} .
$$

Substitute this expression and $u$ for $\pi-u$ in the right-hand side of the equation, it can be found that the equation retains the same. Hence, the interval of $u$ should be $(0, \pi)$.

In addition, this finishes the proof.

Remark 10. It is interesting to write Series (30) in another form:

$$
u=\frac{\pi}{2}-\sum_{n-1}^{\infty} \frac{1}{n} \cos ^{n} u \sin n u
$$

This is a trigonometric expansion of $u$ in the interval $(0, \pi)$, hence it may have a relation to the Fourier series. Indeed, according to the well-known Euler's formula:

$$
e^{i \theta}=\cos \theta+i \sin \theta
$$

we have:

$$
\begin{aligned}
& \cos ^{n} u= \frac{1}{2^{n}}\left(e^{i u}+e^{-i u}\right)^{n}=\frac{1}{2^{n}} \sum_{k=0}^{n}\left(\begin{array}{c}
n \\
k
\end{array}\right) e^{(n-k) i u} e^{-k i u} \\
&= \frac{1}{2^{n}} \sum_{k=0}^{n}\left(\begin{array}{c}
n \\
k
\end{array}\right) e^{(n-2 k) i u} \\
& \sin n u=\frac{1}{2 i}\left(e^{n i u}-e^{-n i u}\right),
\end{aligned}
$$

thus, each factor in the series has the form

$$
\begin{aligned}
\cos ^{n} u \sin n u & =\frac{1}{2^{n+1} i} \sum_{k=0}^{n}\left(\begin{array}{l}
n \\
k
\end{array}\right)\left(e^{(2 n-2 k) i u}-e^{-2 k i u}\right) \\
& =\frac{1}{2^{n+1} i} \sum_{k=1}^{n}\left(\begin{array}{l}
n \\
k
\end{array}\right)\left(e^{2 k i u}-e^{-2 k i u}\right)=\frac{1}{2^{n}} \sum_{k=1}^{n}\left(\begin{array}{l}
n \\
k
\end{array}\right) \sin (2 k u) .
\end{aligned}
$$

If we set

$$
c_{k}=\sum_{n=k}^{\infty} \frac{1}{2^{n}}\left(\begin{array}{l}
n \\
k
\end{array}\right), \text { where } n, k \in N^{+} ;
$$

then Series (32) can be written as

$$
u=\frac{\pi}{2}-\sum_{k=1}^{\infty} c_{k} \sin (2 k u) .
$$

Remark 11. If we put $u=\pi / 4$ in Series (30), a BBP-type formula will be found:

$$
\pi=\sum_{k=0}^{\infty} \frac{1}{(-4)^{k}}\left[\frac{2}{4 k+1}+\frac{2}{4 k+2}+\frac{1}{4 k+3}\right],
$$


which is the same as Formula (13), taking other specific values for $\mathbf{u}$ will not give new BBP-type formulas too. Indeed, Series (30) is deduced from Series (3), thus every numerical result of (30) will be the same as the corresponding numerical result of (3).

Although we cannot obtain more BBP-type formulas from series (3), it is still worthwhile to find new infinite series from it, and these series are themselves remarkable for their simplicity, as the following corollaries.

Corollary 6. Suppose $u \in(0, \pi / 2)$, then

$$
\frac{\pi}{2}=\sum_{n=1}^{\infty} \frac{\sin n u}{n \cos ^{n} u}
$$

Here, $n \in N$.

Proof. In Theorem 1, put

$$
\omega=-x-\frac{1}{x}=-\frac{1}{\sin u \cos u}
$$

then

$$
\arctan (x+\omega)=\arctan \left(-\frac{1}{x}\right)=-\frac{\pi}{2}+\arctan x,
$$

substitute these values in Series (3), and we have

$$
\frac{\pi}{2}=\sum_{n=1}^{\infty} \frac{\sin n u}{n \cos ^{n} u} .
$$

Since this derivation is based on the substitution

$$
\omega=-x-\frac{1}{x}
$$

$x \neq 0$, which means $y \neq 0$. According to the same illustration in Corollary 5 , this series is valid at $u \in(0, \pi / 2)$.

Corollary 7. Suppose $u \in(0, \pi)$, then

$$
\frac{\pi}{2}=\frac{u}{2}+\sum_{n=1}^{\infty} \frac{\sin n u}{n}
$$

Here, $n \in N^{+}$.

Proof. In Theorem 1, put

$$
\omega=-\sqrt{1+x^{2}}
$$

then

$$
\omega=-\frac{1}{\sin u} \text { and } \arctan \left(x-\sqrt{1+x^{2}}\right)=-\frac{1}{2} \arctan \frac{1}{x}=-\frac{u}{2},
$$

substitute these values in Series (3), and we have Series (34). Since this derivation is based on the substitution

$$
\omega=-\sqrt{1+x^{2}}=-\frac{1}{\sin u}
$$

it is apparent that $u \neq 0$, whence $\omega=-\infty$ thus loses its meaning. Furthermore, according to the same illustration in Corollary 5 , it should be $y \in(-\pi / 2, \pi / 2)$. Therefore, $\pi / 2-y=$ $u \in(0, \pi)$.

In addition, this finishes the proof. 
Remark 12. This series is indeed the Fourier expansion of $u$ in interval $(0,2 \pi)$. As it is known, the Fourier series of any function $f(u)$ which satisfies Dirichlet's conditions in interval $(\alpha, \alpha+2 \pi)$ generally has the form:

$$
\frac{a_{0}}{2}+\sum_{m=1}^{\infty}\left(a_{m} \cos m u+b_{m} \sin m u\right),
$$

where $m \in N^{+}$and

$$
\begin{aligned}
& a_{0}=\frac{1}{\pi} \int_{\alpha}^{\alpha+2 \pi} f(u) d u, \\
& a_{m}=\frac{1}{\pi} \int_{\alpha}^{\alpha+2 \pi} f(u) \cos m u d u, \\
& b_{m}=\frac{1}{\pi} \int_{\alpha}^{\alpha+2 \pi} f(u) \sin m u d u .
\end{aligned}
$$

Hence, the coefficients of the Fourier series of $f(u)=u$ (which satisfies Dirichlet's conditions) in the interval $(0,2 \pi)$ will be

$$
\begin{aligned}
& a_{0}=\frac{1}{\pi} \int_{0}^{2 \pi} u d u=2 \pi \\
& a_{m}=\frac{1}{\pi} \int_{0}^{2 \pi} u \cos m u d u=\left[\frac{u \sin u x}{m \pi}\right]_{0}^{2 \pi}+\frac{1}{m \pi} \int_{0}^{2 \pi} \sin m u d u=0 \\
& b_{m}=\frac{1}{\pi} \int_{0}^{2 \pi} u \sin m u d x=-\left[\frac{u \cos m u}{m \pi}\right]_{0}^{2 \pi}+\frac{1}{m \pi} \int_{0}^{2 \pi} \cos m u d u=-\frac{2}{m}
\end{aligned}
$$

Thus, we have the Fourier series:

$$
\frac{u}{2}=\frac{\pi}{2}-\frac{\sin u}{1}-\frac{\sin 2 u}{2}-\frac{\sin 3 u}{3}-\frac{\sin 4 u}{4}-\frac{\sin 5 u}{5}-\ldots
$$

This series is exactly the same as Series (34) in the form. The only difference is, according to Fourier analysis, this formula is valid in the interval $(0,2 \pi)$, but the interval $(0, \pi)$ is according to our method.

Remark 13. If these Series (32), (33), and (34) are differentiated respectively, we obtain new infinite series again:

$$
\begin{gathered}
1+\sum_{n=0}^{\infty} \cos ^{n} u \cos (n+2) u=0 \\
1+\sum_{n=1}^{\infty} \frac{\cos n u}{\cos ^{n} u}=0 \\
\frac{1}{2}+\sum_{n=1}^{\infty} \cos n u=0
\end{gathered}
$$

Here, $n \in N$ in Series (35) and $n \in N^{+}$in Series (36) and (37); these series hold at the same interval with their primitive series, which is Series (32), (33), and (34), respectively.

Remark 14. We shall note that it is interesting and useful to check these series by taking $u$ as specific values, which will immediately show the structure of the series. For instance, put $u=\pi / 4$ in Series (35), and the right side of the equation will be

$$
1-\frac{1}{2}-\frac{1}{2}-\frac{1}{2^{2}}+\frac{1}{2^{3}}+\frac{1}{2^{3}}+\frac{1}{2^{4}}-\frac{1}{2^{5}}-\frac{1}{2^{5}}-\frac{1}{2^{6}}+\frac{1}{2^{7}}+\frac{1}{2^{7}}+\ldots,
$$

or

$$
\begin{gathered}
\left(1+\frac{1}{2^{4}}+\frac{1}{2^{8}}+\ldots\right)-2\left(\frac{1}{2}+\frac{1}{2^{5}}+\frac{1}{2^{9}}+\ldots\right) \\
+2\left(\frac{1}{2^{3}}+\frac{1}{2^{7}}+\frac{1}{2^{11}}+\ldots\right)-\left(\frac{1}{2^{2}}+\frac{1}{2^{6}}+\frac{1}{2^{10}}+\ldots\right),
\end{gathered}
$$

which vanishes apparently. 


\section{Another New Taylor Expansion of $\arctan (x+\omega)$}

In this section, we will give another new Taylor series of $\arctan (x+\omega)$ as a theorem. According to a similar reason as the Remark 11 of Corollary 5, we can not obtain new BBP-type formulas from this new expansion, but the infinite series deduced from it still deserves our attention. For this aim, we need a new lemma as follows:

Lemma 2. Suppose $v=y=\arctan x$, then

$$
\begin{aligned}
& \frac{d^{4 k+1} y}{d x^{4 k+1}}=+(4 k) ! \cos ^{4 k+1} v \cos (4 k+1) v, \\
& \frac{d^{4 k+2} y}{d x^{4 k+2}}=-(4 k+1) ! \cos ^{4 k+2} v \sin (4 k+2) v, \\
& \frac{d^{4 k+3} y}{d x^{4 k+3}}=-(4 k+2) ! \cos ^{4 k+3} v \cos (4 k+3) v, \\
& \frac{d^{4 k+4} y}{d x^{4 k+4}}=+(4 k+3) ! \cos ^{4 k+2} v \sin (4 k+4) v ;
\end{aligned}
$$

Here, $k \in N$.

Proof. We consider to express derivatives of $y$ as functions of $y$ itself this time, but, for clarity, we use $v$ to represent $y$, i.e., $v=\arctan x$. Indeed,

$$
\frac{d y}{d x}=\frac{1}{1+x^{2}}=\frac{1}{1+\tan ^{2} v}=\cos ^{2} v,
$$

or

$$
d y=\cos ^{2} v d x
$$

taking the differential of it, we have

$$
d^{2} y=-\sin 2 v d v d x
$$

since $v=\arctan x$, then $d v=\cos ^{2} v d x$, thus

$$
d^{2} y=-\cos ^{2} v \sin 2 v d x^{2}
$$

Similarly, taking the differential of this formula, we have

$$
d^{3} y=-2 \cos v \cos 3 v d v d x^{2},
$$

substitute $d v$ for $\cos ^{2} v d x$, and it will be

$$
d^{3} y=-2 \cos ^{3} v \cos 3 v d x^{3} .
$$

In the same manner, continuing for higher differentials of $y$, we have

$$
\begin{array}{ll}
\frac{d y}{d x}=\cos v \cos v & \frac{d^{5} y}{d x^{5}}=+4 ! \cos ^{5} v \cos 5 v \\
\frac{d^{2} y}{d x^{2}}=-\cos ^{2} v \sin 2 v & \frac{d^{6} y}{d x^{6}}=-5 ! \cos ^{6} v \sin 6 v \\
\frac{d^{3} y}{d x^{3}}=-2 ! \cos ^{3} v \cos 3 v & \frac{d^{7} y}{d x^{7}}=-6 ! \cos ^{7} v \cos 7 v \\
\frac{d^{4} y}{d x^{4}}=+3 ! \cos ^{4} v \sin 4 v & \frac{d^{8} y}{d x^{8}}=+7 ! \cos ^{8} v \sin 8 v, \ldots ;
\end{array}
$$

Therefore, the proof is also based on induction like Lemma 1.

For $k=1$, Formula (38) is apparently correct.

Suppose

$$
\frac{d^{4 k+1} y}{d x^{4 k+1}}=+(4 k) ! \cos ^{4 k+1} v \cos (4 k+1) v
$$


taking the differential of it, we have

$$
\frac{d^{4 k+2} y}{d x^{4 k+1}}=-(4 k+1) ! \cos ^{4 k} v \sin (4 k+2) v d v
$$

since $d v=\cos ^{2} v d x$, it will be

$$
\frac{d^{4 k+2} y}{d x^{4 k+2}}=-(4 k+1) ! \cos ^{4 k+2} v \sin (4 k+2) v
$$

In the same manner, taking the differential continuously and substitute $d v$ for $\cos ^{2} v d x$, we have

$$
\begin{aligned}
& \frac{d^{4 k+3} y}{d x^{4 k+3}}=-(4 k+2) ! \cos ^{4 k+3} v \cos (4 k+3) v \\
& \frac{d^{4 k+4} y}{d x^{4 k+4}}=+(4 k+3) ! \cos ^{4 k+4} v \sin (4 k+4) v \\
& \frac{d^{4 k+5} y}{d x^{4 k+5}}=+(4 k+5) ! \cos ^{4 k+5} v \sin (4 k+5) v
\end{aligned}
$$

the last one is the same as

$$
\frac{d^{4 k+1} y}{d x^{4 k+1}}=+(4 k+1) ! \cos ^{4 k+1} v \sin (4 k+1) v
$$

In addition, this finishes the proof.

By Lemma 2, substitute Formula (2) into Series (1), and we have another new Taylor expansion of $\arctan (x+\omega)$ as the following theorem.

Theorem 2. Suppose $\omega$ is an arbitrary finite increment of the variable $x$, then

$$
\begin{aligned}
& \arctan (x+\omega)= \arctan x+\sum_{k=0}^{\infty}\left(\frac{\omega^{4 k+1}}{4 k+1} \cos ^{4 k+1} v \cos (4 k+1) v-\frac{\omega^{4 k+2}}{4 k+2} \cos ^{4 k+2} v \sin (4 k+2)\right. \\
&-\left.\frac{\omega^{4 k+3}}{4 k+3} \cos ^{4 k+3} v \cos (4 k+3) v+\frac{\omega^{4 k+4}}{4 k+4} \cos ^{4 k+4} v \sin (4 k+4) v\right) \\
& \text { Here, } k \in N .
\end{aligned}
$$

Corollary 8. Suppose $v \in(-\pi / 2, \pi / 2)$, then

$$
\begin{aligned}
v & =\sum_{n=0}^{\infty}\left(\frac{\sin ^{4 n+1} v \cos (4 n+1) v}{4 n+1}+\frac{\sin ^{4 n+2} v \sin (4 n+2) v}{4 n+2}\right. \\
& \left.-\frac{\sin ^{4 n+3} v \cos (4 n+3) v}{4 n+3}-\frac{\sin ^{4 n+4} v \sin (4 n+4) v}{4 n+4}\right)
\end{aligned}
$$

Here, $n \in N$.

Proof. In Theorem 2, put $\omega=-x$ in Series (39), and we have

$$
\begin{aligned}
v & =\sum_{n=0}^{\infty}\left(\frac{x^{4 n+1}}{4 n+1} \cos ^{4 n+1} v \cos (4 n+1) v+\frac{x^{4 n+2}}{4 n+2} \cos ^{4 n+2} v \sin (4 n+2) v\right. \\
& \left.-\frac{x^{4 n+3}}{4 n+3} \cos ^{4 n+3} v \cos (4 n+3) v-\frac{x^{4 n+4}}{4 n+4} \cos ^{4 n+4} v \sin (4 n+4) v\right)
\end{aligned}
$$

Since $v=\arctan x$, substitute $x=\tan v=\sin v / \cos v$ in Series (41), and we obtain Series (40). According to the same illustration in the Corollary 5, it should be $y \in(0, \pi / 2)$, thus $v \in(0, \pi / 2)$ because $v=y$. In addition, it can be easily seen that the equation in this corollary stays the same when we substitute $v$ for $-v$, thus the interval of $v$ should be $(-\pi / 2, \pi / 2)$.

In addition, this finishes the proof. 
Corollary 9. Suppose $v \in(0, \pi / 2)$, then

$$
\frac{\pi}{2}=\sum_{n=0}^{\infty}\left(\frac{\cos (4 n+1) v}{(4 n+1) \sin ^{4 n+1} v}+\frac{\sin (4 n+2) v}{(4 n+2) \sin ^{4 n+2} v}-\frac{\cos (4 n+3) v}{(4 n+3) \sin ^{4 n+3} v}-\frac{\sin (4 n+4) v}{(4 n+4) \sin ^{4 n+4} v}\right)
$$

Here, $n \in N$.

Proof. In Theorem 2, put

$$
\omega=-x-\frac{1}{x}=-\frac{1}{\sin v \cos v}
$$

then

$$
\arctan (x+\omega)=\arctan \left(-\frac{1}{x}\right)=-\frac{\pi}{2}+\arctan x,
$$

substitute these values in Series (39), and we obtain Series (42). According to the same illustration in the proof of Corollary 6 , it should be $y \in(0, \pi / 2)$, thus $v \in(0, \pi / 2)$ because $v=y$.

This finishes the proof.

Corollary 10. Suppose $v \in(-\pi / 2, \pi / 2)$, then

$$
\frac{\pi}{4}=-\frac{v}{2}+\sum_{n=0}^{\infty}\left(\frac{\cos (4 n+1) v}{4 n+1}+\frac{\sin (4 n+2) v}{4 n+2}-\frac{\cos (4 n+3) v}{4 n+3}-\frac{\sin (4 n+4) v}{4 n+4}\right)
$$

Here, $n \in N$.

Proof. In Theorem 2, put

$$
\omega=-\sqrt{1+x^{2}}
$$

since now

$$
\omega=-\frac{1}{\cos v} \text { and } \arctan \left(x-\sqrt{1+x^{2}}\right)=-\frac{1}{2} \arctan \frac{1}{x}=\frac{v}{2}-\frac{\pi}{4}
$$

substituting these values in Series (39), and we obtain Series (43). According to the same illustration in the proof of Corollary 7 , it should be $y \in(-\pi / 2, \pi / 2)$, thus $v \in(-\pi / 2, \pi / 2)$ because $v=y$.

Remark 15. It is interesting and maybe curious rather than useful to note that Leibniz's $\pi / 4$ formula can also be obtained from this series, by setting $v=0$.

Remark 16. Take the differentials of Series (40), (42), (43), respectively, and we have new series again:

$$
\begin{gathered}
1=\sum_{n=0}^{\infty}\left(\sin ^{4 n} v \cos (4 n+2) v+\sin ^{4 n+1} v \sin (4 n+3) v\right. \\
\left.-\sin ^{4 n+2} v \cos (4 n+4) v-\sin ^{4 n+3} v \sin (4 n+5) v\right) \\
0=\sum_{n=0}^{\infty}\left(\frac{\cos (4 n) v}{\sin ^{4 n+2} v}+\frac{\sin (4 n+1) v}{\sin ^{4 n+3} v}-\frac{\cos (4 n+2) v}{\sin ^{4 n+4} v}-\frac{\sin (4 n+3) v}{\sin ^{4 n+5} v}\right) \\
-\frac{1}{2}=\sum_{n=0}^{\infty}(\sin (4 n+1) v-\cos (4 n+2) v-\sin (4 n+3) v+\cos (4 n+4) v)
\end{gathered}
$$

Here, $n \in N$, these series are valid at the same intervals with their primitive series, i.e., Series (40), (42), and (43), respectively. 


\section{Conclusions}

In this study, we propose two new Taylor expansions of $\arctan (x+\omega)$. These new expansions have clear structure and exhibit certain law of progression. We obtain several remarkable infinite series from them by specific substitutions. These infinite series give new BBP-type formulas.

It is known that the BBP-type formula was found firstly by using the PSLQ algorithm. However, this is not the only approach. Indeed, this numerical method is not priori because it can only provide the formula as a result but not reveal the origin of it. For this reason, when a BBP formula was discovered by algorithm, we still need a mathematical proof to make the conclusion rigorous. The second approach, which is also what we adopted, is to find infinite series firstly, and their numerical results give BBP-type formulas. Thus, this approach is priori, and it is generally based on calculus. It is significant and useful to realize the difference of these two approaches. In addition, it should be noted that the inverse tangent function is generally related to the second approach. Indeed, Gregory's series can be seen as the simplest series of this type, and it is the infinite series of $\arctan x$, when $x=1$; this series gives Leibniz's formula.

Author Contributions: Conceptualization, X.W.; methodology, X.W.; validation, X.W., Z.C. and Y.Z.; formal analysis, X.W. and Y.Z.; investigation, X.W.; resources, X.W.; writing-original draft preparation, X.W. and Y.Z.; writing-review and editing, X.W. and Y.Z.; visualization, X.W., Z.C. and Y.Z.; supervision, Z.C. and Y.Z. All authors have read and agreed to the published version of the manuscript.

Funding: This research received no external funding.

Institutional Review Board Statement: Not applicable.

Informed Consent Statement: Not applicable.

Data Availability Statement: Not applicable.

Conflicts of Interest: The authors declare no conflict of interest.

\section{References}

1. Roy, R. The Discovery of the Series Formula for $\pi$ by Leibniz, Gregory and Nilakantha. Math. Mag. 1990, 63, 291-306. [CrossRef]

2. Euler, L. Introduction to Analysis of the Infinite; Springer: New York, NY, USA, 1988.

3. Euler, L. De summis serierum reciprocarum. Comment. Acad. Sci. Petropolitanae. 1740, 7, 123-134.

4. Euler, L. Institutiones Calculi Differentialis Cum Eius usu in Analysi Finitorum ac Doctrina Serierum; Academiae Imperialis Scientiarum Petropolitanae: London, UK, 1755; Volume 2, chapter III.

5. Euler, L. Institutionum Calculi Integralis Volumen Primum; Academiae Imperialis Scientiarum Petropolitanae: London, UK, 1768; Volume 1, chapter III.

6. Euler, L. De variis modis circuli quadraturam numeris proxime exprimendi. Comment. Acad. Sci. Petropolitanae 1744, 9, 222-236.

7. Euler, L. Demonstration de la somme de cette suite $1+1 / 4+1 / 9+1 / 16+\ldots$ J. Litt. D'allemagne Suisse Du Nord 1743, 2, 115-127.

8. Bailey, D.H.; Borwein, P.; Plouffe, S. On the rapid computation of various polylogarithmic constants. Math. Comp. 1997, 66, 903-913. [CrossRef]

9. Adamchik, V.; Wagon, S. A Simple Formula for Pi. Amer. Math. Mon. 1997, 104, 852-855. [CrossRef]

10. Chan, H.C. $\pi$ in terms of $\varphi$. Fibonacci Quart. 2006, 44, 141-144.

11. Adegoke, K. A non-PSLQ route to BBP-type formulas. arXiv 2016, arXiv:10.5539/jmr.v2n2p56. [CrossRef]

12. Adegoke, K. Symbolic routes to BBP-type formulas of any degree in arbitrary bases. Appl. Math. Inf. Sci. 2011, 5, $264-275$.

13. Bailey, D.H.; Borwein, J. Experimental mathematics: Examples, methods and implications. Not. Amer. Math. Soc. 2005, 52, 502-514.

14. Bailey, D.H.; Borwein, J.; Borwein, P.; Plouffe, S. The quest for pi. Math. Intelligencer. 1997, 19, 50-57. [CrossRef]

15. Chan, H.C. More formulas for $\pi$. Amer. Math. Mon. 2006, 113, 452-455. [CrossRef]

16. Chan, H.C. Machin-type formulas expressing $\pi$ in terms of $\varphi$. Fibonacci Quart. 2008, 46, 32-37.

17. Wei, C. Several BBP-type formulas for $\pi$. Integral Transform. Spec. Funct. 2015, 26, 315-324. [CrossRef]

18. Zhang, W. New formulae of BBP-type with different moduli. J. Math. Anal. Appl. 2013, 398, 46-60. [CrossRef]

19. Zhang, W.; Zhang, Y. New formulae of BBP-type modulo 6. Quaest. Math. 2014, 37, 393-400. [CrossRef]

20. Guillera, J. History of the formulas and algorithms for $\pi$. Gac. R. Soc. Mat. Esp. 2007, 10, 159-178.

21. Takahashi, D. On the computation and verification of $\pi$ using BBP-type formulas. Ramanujan J. 2020, 51, 177-186. [CrossRef] 
22. Takahashi, D. On the use of Montgomery multiplication in the computation of binary BBP-type formulas for mathematical constants. Ramanujan J. 2021, 51, 1-9. [CrossRef]

23. Takahashi, D. Computation of the 100 quadrillionth hexadecimal digit of $\pi$ on a cluster of Intel Xeon Phi processors. Parallel Comput. 2018, 75, 1-10. [CrossRef]

24. Karrels, E. Computing digits of $\pi$ with CUDA. 2017. Available online: http:/ / www.karrels.org/pi/ (accessed on 31 October 2021).

25. Bailey, D.H.; Crandall, R. On the Random Character of Fundamental Constant Expansions. Exp. Math. 2001, 10, 175-190. [CrossRef]

26. Bailey, D.H. A compendium of BBP-type formulas for mathematical constants. 2017, pp. 1-38, Preprint. Available online: https://www.davidhbailey.com/dhbpapers/bbp-formulas.pdf (accessed on 31 October 2021). 